\title{
Avaliação e desenvolvimento de ambiente virtual de aprendizagem no ensino da disciplina de Diagnóstico Oral por meio do blended learning
}

\author{
Ana Cristina Zuzarte Ferreira Santos*; Isabella Kaynara Ribeiro de Andrade**; Marta Rabello \\ Piva***; Wilton Mitsunari Takeshita****
}

\author{
* Cirurgiã-dentista graduada pela Universidade Federal de \\ Sergipe \\ ** Estudante de Odontologia da Universidade Federal de \\ Sergipe \\ *** Doutora em Patologia Oral, professora da disciplina de \\ Patologia Oral do Departamento de Odontologia da \\ Universidade Federal de Sergipe \\ **** Professor Doutor da disciplina de Radiologia Odontológica e \\ Diagnóstico Oral do Departamento de Odontologia da \\ Universidade Federal de Sergipe
}

Recebido em 19/12/2016. Aprovado em 05/04/2017.

\begin{abstract}
RESUMO
O Blended Learning (B-learning) combina ensino a distância com ensino presencial em sala de aula. A plataforma Moodle é um Sistema de Gestão da Aprendizagem (SGA), que funciona baseado na construção da aprendizagem a partir da interação, e ainda oferece ferramentas que favorecem a integração das atividades presenciais com atividades à distância, mas de fácil usabilidade. O objetivo deste estudo foi avaliar e ampliar os cenários de aprendizagem por meio da criação de um ambiente virtual como material didático de apoio, com intuito de transformá-lo em ferramenta no processo de educação para estudantes, para isso foram selecionados 13 alunos da graduação, que já tinham cursado a disciplina Diagnostico Oral da Universidade Federal de Sergipe (UFS). Ao final do curso, os voluntários foram submetidos a um questionário para avaliar o AVA métodos especializados por imagem - diagnostico oral (AVA-DO), quanto ao material de apoio disponibilizado no ambiente, bem como a acessibilidade e usabilidade no AVA-DO, e por fim, o ganho de conhecimento do aluno após a experiência na educação a distância. A avaliação do ganho de aprendizado do estudante foi feito por meio de questionário a respeito do material ensinado. Todas as questões tiveram mais $80 \%$ de acertos. Os resultados expõem também a dificuldade do aluno em manusear o Moodle, encontrando obstáculos na usabilidade e acessibilidade da ferramenta. Conclui-se o emprego do ambiente virtual de aprendizagem favoreceu o processo de aprendizagem dos estudantes de graduação, consistindo em mais um cenário de aprendizagem para a Odontologia.
\end{abstract}

Descritores: Educação a Distância. Aprendizagem. Radiologia. 


\section{INTRODUÇÃO}

A educação a distância (EaD) apresenta-se como um grande passo para a democratização do conhecimento intelectual, oportunizando o acesso ao ensino de forma mais fácil, democratizando o conhecimento e facilitando a aprendizagem ao utilizar atividades teóricas e práticas que possam ser realizadas a partir de orientações remotas $^{1,2}$.

No ensino superior, de um modo geral, a oferta da educação a distância $(\mathrm{EaD})$ atrela-se à necessidade de atender a demandas da sociedade, mais especificamente àquelas que dizem respeito ao mundo do trabalho, no sentido de concretizar, de modo rápido e flexível, a preparação de profissionais, seja em termos de formação inicial ou continuada . No Brasil somente em 1923, por iniciativa da Rádio Roquete Pinto, o EaD é utilizada no ensino de cidadania aos ouvintes. A chegada do rádio e, posteriormente da televisão, provocou uma revolução nessa modalidade educacional e com a criação das TVs Educativas, em 1965, a televisão teria uma penetração maior na formação da sociedade brasileira ${ }^{3}$. Com um crescimento extraordinário e acessado por milhares de usuários, o $\mathrm{EaD}$ é uma realidade presente em praticamente todas as instituições de ensino superior no Brasil.

Segundo Belloni", o "EaD aparece, cada vez mais, no contexto das sociedades contemporâneas, como uma modalidade de educação adequada para atender às novas demandas educacionais decorrentes das mudanças na nova ordem econômica mundial".

Neste contexto, o rápido desenvolvimento tecnológico auxilia todas as profissões no planejamento, execução e conclusão de quaisquer serviços. Em todo o mundo, um número incontável de informações é digitalizado, bancos de dados são gerados e estatísticas surgem a partir do simples clique em um botão a cada segundo que passa. As imagens apresentam papel fundamental no universo da Teleodontologia. Como instrumentos de imagem utilizados como apoio na teleodontologia temos por exemplo, o scanner com dispositivo para transparência ${ }^{5,6}$, as máquinas fotográficas digitais $^{7}$, a radiografia digital ${ }^{7-10}$ e as câmeras de vídeo $^{11}$.

A Odontologia é uma ciência complexa, não exata e fragmentada em diferentes áreas de atuação, sendo que essa complexidade também acompanhada seu processo educativo ${ }^{12}$. Além de conhecer o conteúdo teórico, é necessário que o estudante o coloque em prática priorizando a melhoria da qualidade de vida de uma determinada população ${ }^{13}$. Assim, o estudante de odontologia necessita correlacionar os conhecimentos científicos adquiridos em disciplinas básicas à vivência clínica, para que possa entender o processo de construção do diagnóstico e desta maneira aprender a indicar e a planejar o melhor tratamento, melhorando o prognóstico e a qualidade de vida do paciente. Por isso, para tornar possível o EaD na Odontologia pode-se implementar o blended learning (B-leerning), aprendizado híbrido, ou seja, a educação presencial combinada com material de apoio complementar ${ }^{14}$.

O estudante de Odontologia encontra dificuldade em aplicar os conhecimentos teóricos adquiridos em disciplinas como Patologia Oral, Radiologia e Estomatologia à prática clínica. Essa dificuldade é mais evidente quando é necessário correlacionar achados microscópicos e radiográficos aos dados macroscópicos coletados no exame clínico, sendo essa correlação fundamental para a elaboração e entendimento do diagnóstico ${ }^{15}$. $\mathrm{O}$ processo ensino-aprendizagem constitui o fundamento da prática docente universitária. No entanto, paralelamente ou como parte inseparável desse processo, a avaliação é a ferramenta que fornece informações oportunas que buscam investigar se 
a aprendizagem está sendo alcançada, bem como indica a retomada do caminho que busca o objetivo final $^{16}$.

A construção de um correto diagnóstico é pré-requisito para elaboração e execução de um plano de tratamento adequado, e o domínio dos conteúdos específicos é essencial para a prática profissional, para que desta forma a saúde do paciente possa ser reestabelecida. Assim, no processo educativo em Odontologia, o professor precisa orientar o aluno a buscar os conhecimentos teóricos para desenvolver uma prática clínica consciente e cumprir sua função social $^{13,17}$.

Nesse novo ambiente de trabalho da modalidade $\mathrm{EaD}$, o estudante é convidado a refletir sobre o tema proposto, sem receber o conteúdo de maneira passiva, e assim ter subsídios para construir seu próprio saber e desenvolver técnicas individuais de apreensão do conhecimento $^{18,19}$.

\section{A plataforma Moodle (Modular Object} Oriented Dynamic Learning Environment) é Sistema de Gestão da Aprendizagem (SGA) criado a partir de uma concepção construtivista, que se fundamenta na construção da aprendizagem a partir da interação, por ser de utilização gratuita, esse modelo de plataforma tem sido amplamente utilizado, no entanto existem outros tipos de sistemas e também mais modernos. O SIGAA (Sistema Integrado de gestão em atividades acadêmicas) permite essa logística, pois é um sistema que informatiza os procedimentos da área acadêmica, incluindo atividades de ensino a distância e ainda disponibiliza um ambiente virtual de aprendizagem. O Moodle apresenta ferramentas que favorecem a integração das atividades presenciais com atividades vinculadas ao $\mathrm{EaD}^{16}$, permitindo ao aluno o acesso aos conteúdos expostos em sala de aula, participação de fórum de discussão, resolução de questões.
O objetivo deste estudo foi avaliar e ampliar os cenários de aprendizagem por meio da criação de um ambiente virtual (AVA-DO) como material didático de apoio, com intuito de se transformar numa ferramenta no processo de construção do conhecimento para os estudantes de Odontologia, ampliando os cenários de aprendizagem e permitindo a reflexão sobre os processos de aprendizagem.

\section{MATERIAIS E MÉTODOS}

A pesquisa foi aprovada pelo Comitê de Ética em Pesquisa Envolvendo Seres Humanos da Universidade Federal de Sergipe (CAAE no. 51151815.2.0000.5546). Tratou-se de um estudo observacional com delineamento transversal e abordagem quantitativa descritiva. Para tanto, participaram do estudo 13 alunos da graduação em Odontologia, que finalizaram a disciplina Diagnostico Oral, concordaram em participar e assinaram um Termo de Consentimento Livre e Esclarecido.

$\mathrm{O}$ ambiente virtual foi desenvolvido no SIGAA, que é a plataforma Moodle da UFS com conteúdo programático previamente definido e dividido em dois tópicos: 1) Apresentação do ambiente virtual; 2) Aprendendo a utilizar software CS 3D IMAGING para Tomografia Computadorizada de Feixe Cônico (TCFC) (figuras 1 e 2).

$\mathrm{O}$ material didático adicionado no ambiente virtual de aprendizado foi um tutorial de autoria própria sobre o software CS 3D IMAGING, o qual orienta o estudante quanto à instalação do programa e à manipulação de imagens tomográficas (figuras 3 e 4).

$\mathrm{O}$ ambiente virtual de aprendizado foi aberto somente para os alunos voluntários que se propuseram a participar da pesquisa, por meio da assinatura do Termo de Consentimento Livre e Esclarecido. No curso, o conteúdo foi exposto em partes, visto que primeiramente foi lançado um 
tópico sobre apresentação do AVA-DO, nele havia informações sobre o mapa do ambiente virtual, sinalizando a localização das seções disponíveis na plataforma e como utilizá-las. No segundo momento foi disponibilizado um tutorial ensinando ao estudante como instalar o software CS 3D IMAGING, expondo todas as etapas de forma ilustrada e outro fazendo uma explanação sobre como manipular imagens tomográficas no software, utilizando também a ilustração como método explicativo.

No último momento do estudo, os voluntários foram submetidos a um questionário de avaliação disponível na seção enquetes do AVA-DO (figura 5), contendo questionamentos sobre o ganho de conhecimento após a experiência no AVA, com perguntas elaboradas a partir do conteúdo programático exposto no AVA-DO. Em adição, quesitos sobre a contribuição do material de apoio disponibilizado ao processo de ensino-aprendizagem, questionando se as imagens existentes no treinamento contribuíram na sua aprendizagem, se a forma como era disposto o conteúdo facilitou o processo de ensino-aprendizagem e por fim, se o formato dos tutoriais disponibilizados no AVA-DO estimulou a aprendizagem.
Também foi avaliada a acessibilidade à plataforma, nesse momento o aluno era convidado a refletir sobre a facilidade de acesso ao ambiente virtual, sobre o acesso aos textos de apoio disponibilizados, e se era possível fazer download dos arquivos disponibilizados. Por fim, a última parte do questionário permitia ao aluno avaliar o AVA-DO de acordo com a usabilidade, indagando sobre a facilidade de encontrar as informações desejadas e de como era fácil a navegação entre as diversas seções/aulas do treinamento.

Os dados foram tabulados em planilha do Microsoft Office Excel (2007) e registrados na forma de banco de dados no programa SPSS 17.0 (SPSS Inc., Chicago, EUA) e utilizado o programa Minitab 14.2 (MinitabInc, Pensilvânia, EUA) para a análise quantitativa e aplicação dos testes estatísticos. Para os procedimentos descritivos os dados foram apresentados em porcentagens. Os dados foram analisados por meio de tabelas de distribuição de frequência. Para comparar estatisticamente as repostas, foram realizados os procedimentos de inferência estatística, com base em teste não-paramétrico Qui-quadrado para proporções consideradas iguais $(\mathrm{p}<0,05)$.

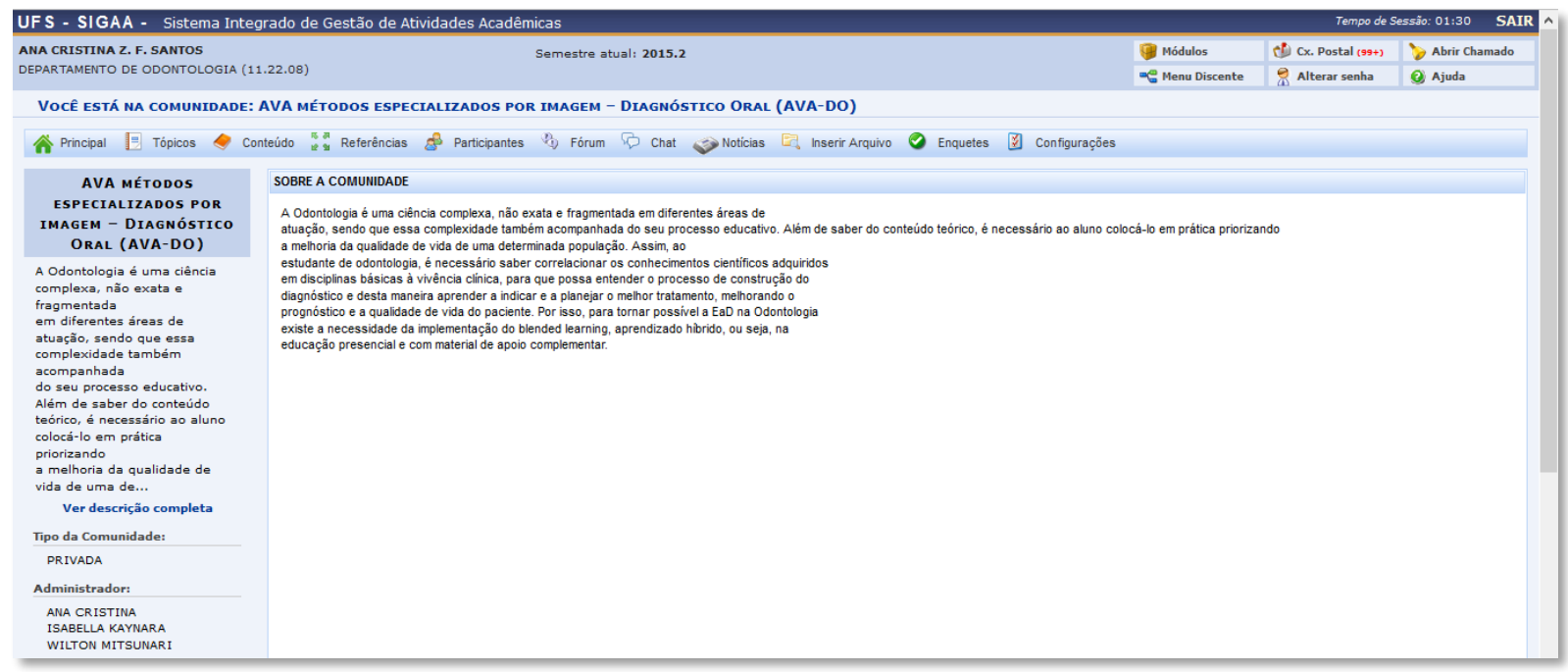

Figura 1 - Apresentação do AVA-DO 


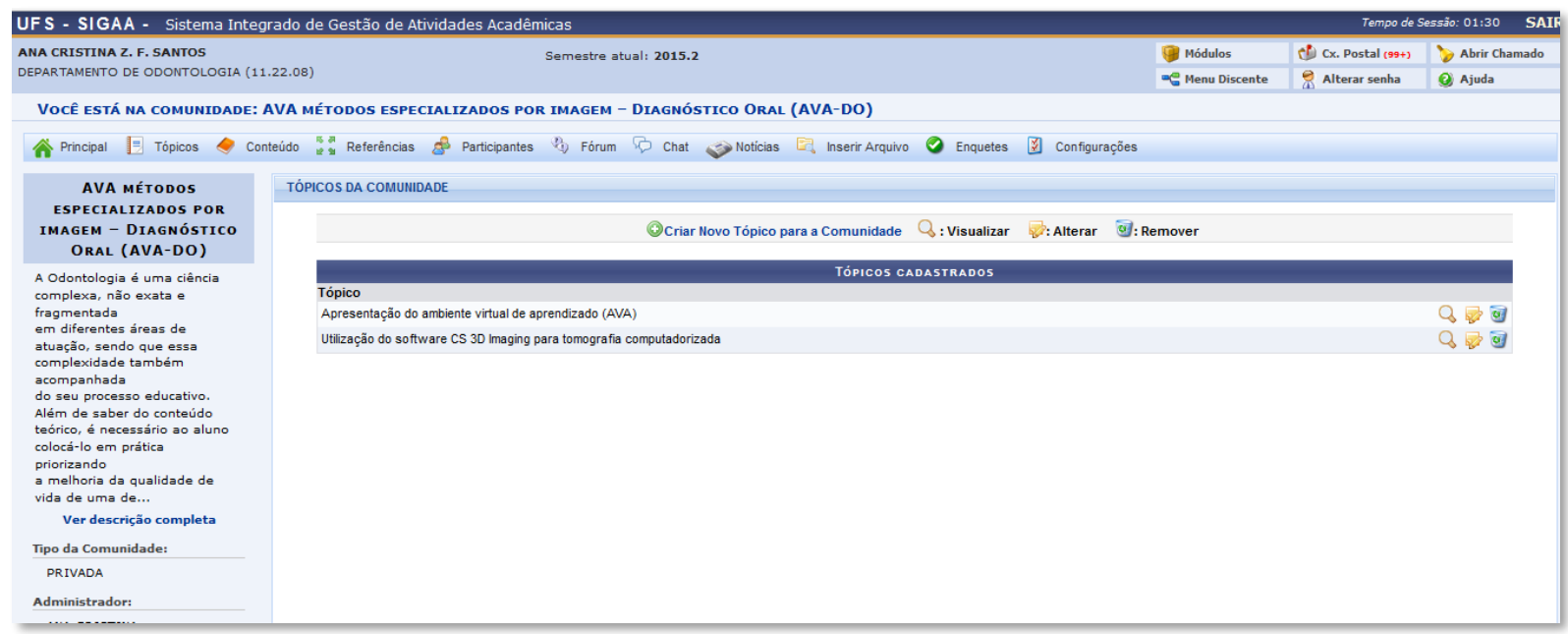

Figura 2 - Tópicos propostos no ambiente virtual de aprendizagem

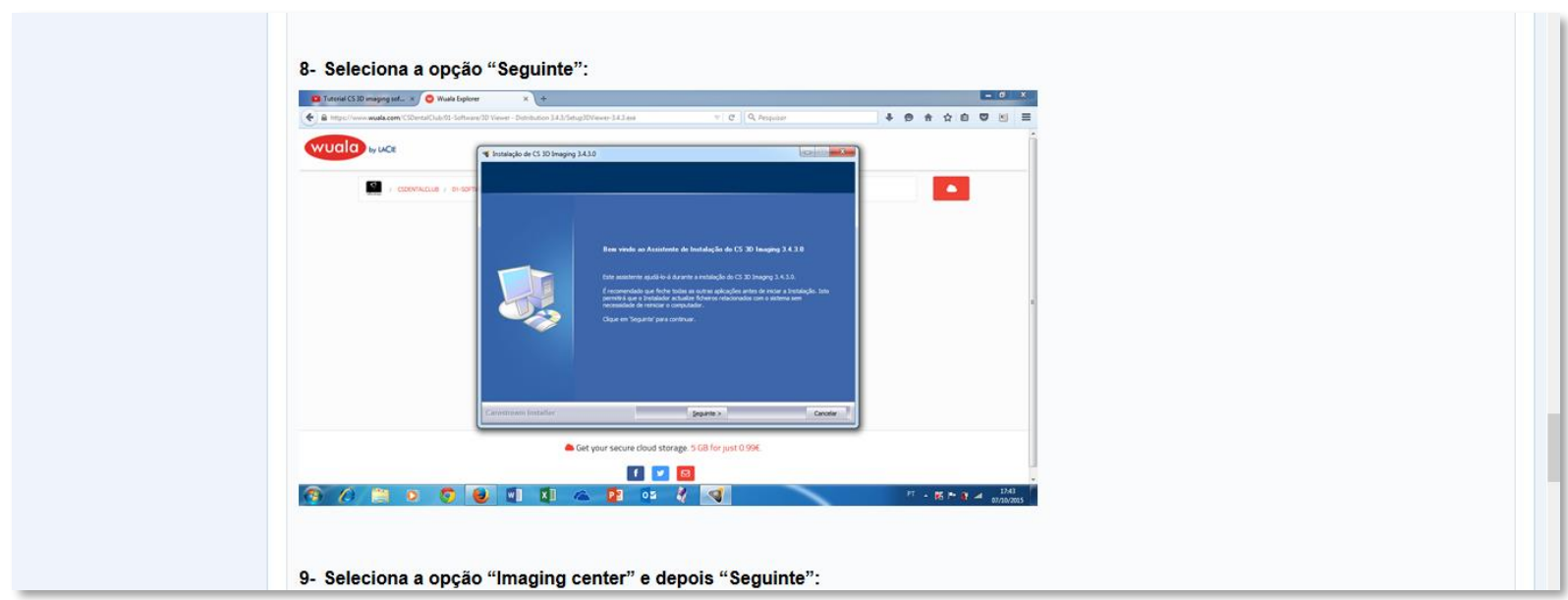

Figura 3 - Conteúdo programático. Tutorial de instalação do software CS 3D IMAGING

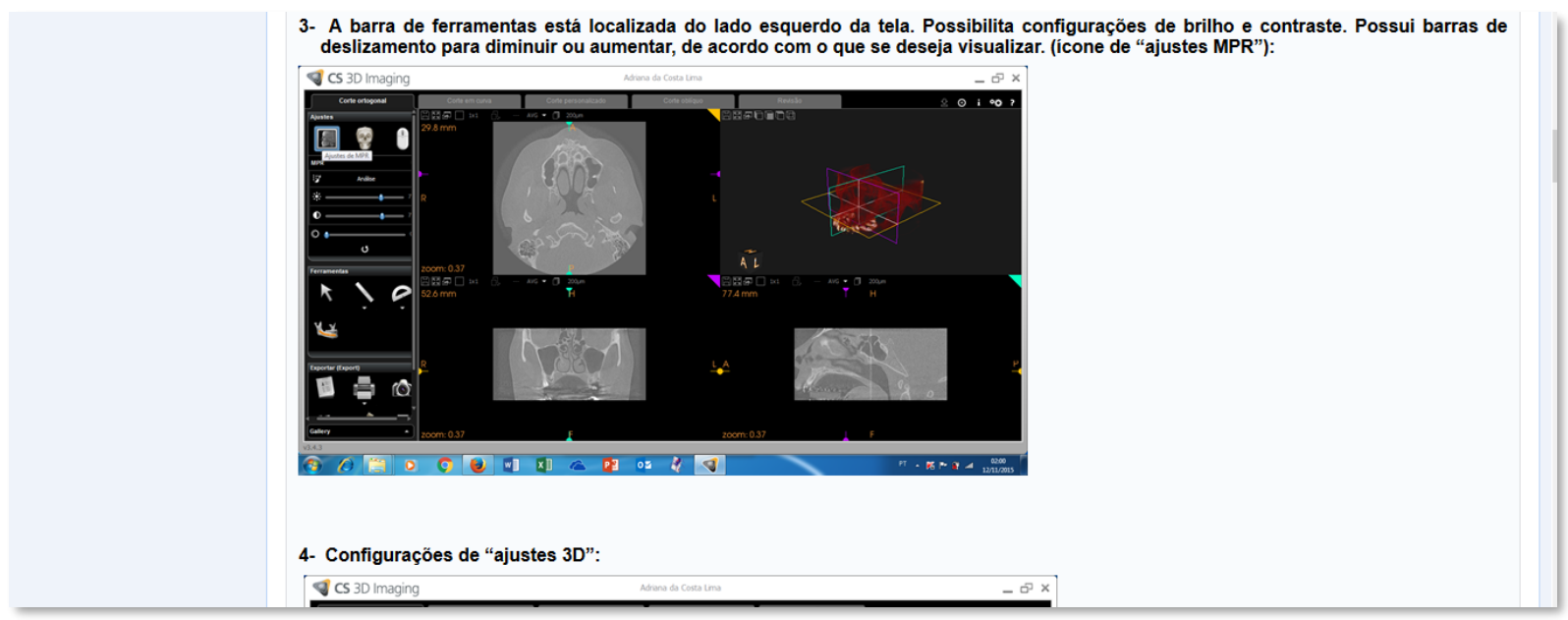

Figura 4 - Conteúdo programático. Tutorial de uso básico do software CS 3D IMAGING 
Avaliação e desenvolvimento de ambiente virtual de aprendizagem no ensino da disciplina de Diagnóstico Oral

\begin{tabular}{|c|c|}
\hline \multicolumn{2}{|l|}{$\begin{array}{ll}\text { ENQUETES CADAstrados } & \text { Casto }\end{array}$} \\
\hline Pergunta da Enquete & Criador da Enquete \\
\hline Quanto à ac essibilidade ao ambiente AVA-DO: 1 . Você tinha facilidade de ac esso ao ambiente virtual? & $\begin{array}{l}\text { ANA CRISTINA ZUZARTE } \\
\text { FERREIRA SANTOS }\end{array}$ \\
\hline Quanto à ac essibilidade ao ambiente AVA-DO: 2 . Você tinha ac esso fácil aos textos de apoio disponibilizados no AVA-DO? & $\begin{array}{l}\text { ANA CRISTINA ZUZARTE } \\
\text { FERREIRA SANTOS }\end{array}$ \\
\hline Quanto à ac essibilidade ao ambiente AVA-DO: 3 . Era possivel fazer download dos arquivos disponibilizados no AVA-DO? & $\begin{array}{l}\text { ANA CRISTINA ZUZARTE } \\
\text { FERREIRA SANTOS }\end{array}$ \\
\hline Quanto à navegabilidade no ambiente AVA-DO: 1 . Você tinha fac ilidade de encontrar as informações desejadas? & $\begin{array}{l}\text { ANA CRISTINA ZUZARTE } \\
\text { FERREIRA SANTOS }\end{array}$ \\
\hline Quanto à navegabilidade no ambiente AVA-DO: 2 . Você tinha fac ilidade de navegação entre as diversas seções/aulas do treinamento? & $\begin{array}{l}\text { ANA CRISTINA ZUZARTE } \\
\text { FERREIRA SANTOS }\end{array}$ \\
\hline $\begin{array}{l}\text { Quanto ao aprendizado: De acordo com as movimentações o Eixo azul possibilita movimentos na vertic al para direita e esquerda, porém não pode ser } \\
\text { angulado. }\end{array}$ & $\begin{array}{l}\text { ANA CRISTINA ZUZARTE } \\
\text { FERREIRA SANTOS }\end{array}$ \\
\hline Quanto ao aprendizado: É possivel no 'corte ortogonal' a visualização em 3D: & $\begin{array}{l}\text { ANA CRISTINA ZUZARTE } \\
\text { FERREIRA SANTOS }\end{array}$ \\
\hline $\begin{array}{l}\text { Quanto ao aprendizado: É possível realizar movimentos das diferentes vistas a partir de eixos identific ados por cores. } 0 \text { amarelo corresponde a vista } \\
\text { sagital, possibilitando movimentos para cima e para baixo. }\end{array}$ & $\begin{array}{l}\text { ANA CRISTINA ZUZARTE } \\
\text { FERREIRA SANTOS }\end{array}$ \\
\hline Quanto ao aprendizado: Na caixa de ferramentas tem as opções realizar medições ou angulações, segundo a esc ala de: & $\begin{array}{l}\text { ANA CRISTINA ZUZARTE } \\
\text { FERREIRA SANTOS }\end{array}$ \\
\hline $\begin{array}{l}\text { Quanto ao aprendizado: No "corte em curva", em ferramentas possui um ic one que possibilita criar a arcada manualmente para obtenção de uma vista } \\
\text { panorâmic a. Para isso seleciona a área que deseja de acordo com a vista } 3 \mathrm{D} \text { no plano axial (eixo roxo). }\end{array}$ & $\begin{array}{l}\text { ANA CRISTINA ZUZARTE } \\
\text { FERREIRA SANTOS }\end{array}$ \\
\hline Quanto ao aprendizado: O roxo corresponde a vista coronal, possibilitando movimentos no sentido anteroposterior. & $\begin{array}{l}\text { ANA CRISTINA ZUZARTE } \\
\text { FERREIRA SANTOS }\end{array}$ \\
\hline Quanto ao aprendizado: $O$ software possibilita a aquisição de imagens em quais tipos de cortes? & $\begin{array}{l}\text { ANA CRISTINA ZUZARTE } \\
\text { FERREIRA SANTOS }\end{array}$ \\
\hline Quanto ao material de apoio disponibilizado: 1 . Você considera que as imagens existentes no treinamento contribuiram na sua aprendizagem? & $\begin{array}{l}\text { ANA CRISTINA ZUZARTE } \\
\text { FERREIRA SANTOS }\end{array}$ \\
\hline Quanto ao material de apoio disponibilizado: 2 . A forma como era disposto o conteúdo fac ilitou o processo de ensino-aprendizagem? & $\begin{array}{l}\text { ANA CRISTINA ZUZARTE } \\
\text { FERREIRA SANTOS }\end{array}$ \\
\hline $\begin{array}{l}\text { Quanto ao material de apoio disponibilizado: } 3 \text {. O Formato dos tutoriais disponibilizados no AVA métodos especializados por imagem - Diagnóstico Oral } \\
\text { (AVA-DO) estimulou a aprendizagem? }\end{array}$ & $\begin{array}{l}\text { ANA CRISTINA ZUZARTE } \\
\text { FERREIRA SANTOS }\end{array}$ \\
\hline
\end{tabular}

Figura 5 - Questionário final disponível na seção enquetes

\section{RESULTADOS}

De acordo com metodologia do presente trabalho foram obtidos os seguintes resultados apresentados nas tabelas 1 a 4 . As enquetes foram subdivididas em blocos de questões com destaque para os resultados, a seguir descritos:

Quanto à acessibilidade ao ambiente AVADO, $43 \%$ dos voluntários tiveram dificuldades de acesso ao conteúdo do ambiente virtual e $58 \%$ apresentaram dificuldade de acesso aos textos de apoio disponível (tabela 1).

Quanto à usabilidade no ambiente AVADO, $50 \%$ dos voluntários sinalizaram dificuldades na navegação entre as diversas seções/aulas do treinamento (tabela 2).

Com relação ao aprendizado, nas questões específicas sobre o conteúdo programático, todas as questões tiveram mais $80 \%$ de acertos (tabela
3). Quanto ao material de apoio disponibilizado, $100 \%$ dos alunos consideraram que o treinamento contribuiu para a aprendizagem (tabela 4).

\section{DISCUSSÃO}

A tecnologia tem possibilitado à área de educação a abertura de uma gama de possibilidades no que diz respeito aos recursos educacionais, abrindo as portas para a flexibilização de espaço e tempo de ensino, democratização do conhecimento, facilitação do processo ensino-aprendizagem. Aliar as novas tecnologias aos processos e atividades educativos é algo que pode significar dinamismo, promoção de novos e constantes conhecimentos e, além disso, proporcionar uma interatividade, superando as distâncias territoriais ${ }^{20}$. 
Tabela 1 - Acessibilidade ao ambiente AVA-DO

\begin{tabular}{lcccc}
\hline \multicolumn{1}{c}{ Pergunta } & \multicolumn{4}{c}{ Respostas (\%) } \\
\cline { 2 - 5 } & Sempre & Frequentemente & Às vezes & Nunca \\
\hline $\begin{array}{l}\text { Você tinha facilidade de acesso } \\
\text { ao ambiente virtual? }\end{array}$ & $22 \%$ & $8 \%$ & $29 \%$ & $43 \%$ \\
$\begin{array}{l}\text { Você tinha acesso fácil aos } \\
\begin{array}{l}\text { textos de apoio disponibili- } \\
\text { zados no AVA-DO? }\end{array}\end{array}$ & $8 \%$ & $2 \%$ & $3 \%$ & $58 \%$ \\
$\begin{array}{l}\text { Era possível fazer download } \\
\text { dos arquivos disponibiliza- } \\
\text { dos no AVA-DO? }\end{array}$ & $47 \%$ & $16 \%$ & $16 \%$ & $24 \%$ \\
\hline
\end{tabular}

Tabela 2 - Navegabilidade no ambiente AVA-DO

\begin{tabular}{lcccc}
\hline \multicolumn{1}{c}{ Pergunta } & \multicolumn{4}{c}{ Respostas (\%) } \\
\cline { 2 - 5 } & Sempre & Frequentemente & Às vezes & Nunca \\
\hline $\begin{array}{l}\text { Você tinha facilidade de } \\
\text { encontrar as informações } \\
\text { desejadas? }\end{array}$ & $29 \%$ & $0 \%$ & $36 \%$ & $36 \%$ \\
$\begin{array}{l}\text { Você tinha facilidade de } \\
\text { navegação entre as diversas } \\
\text { seções/aulas do treinamento? }\end{array}$ & $36 \%$ & $0 \%$ & $15 \%$ & $50 \%$ \\
\hline
\end{tabular}

Tabela 3 - Quanto ao aprendizado: Questões especificas relacionadas a utilização do software CS 3D IMAGING

\begin{tabular}{|c|c|c|c|}
\hline \multirow[t]{2}{*}{ Pergunta } & \multicolumn{3}{|c|}{ Respostas (\%) } \\
\hline & Certo & Errado & Valor de p \\
\hline $\begin{array}{l}\text { O Eixo azul possibilita movimentos na vertical para direita e } \\
\text { esquerda, porém não pode ser angulado. }\end{array}$ & $85 \%$ & $15 \%$ & $* \mathrm{p}<0,001$ \\
\hline É possível no “corte ortogonal” a visualização em 3D & $100 \%$ & $0 \%$ & $\mathrm{n} / \mathrm{a}$ \\
\hline $\begin{array}{l}\text { É possível realizar movimentos das diferentes vistas a partir de } \\
\text { eixos identificados por cores. O amarelo corresponde a vista } \\
\text { sagital, possibilitando movimentos para cima e para baixo. }\end{array}$ & $90 \%$ & $10 \%$ & $* \mathrm{p}<0,001$ \\
\hline $\begin{array}{l}\text { Na caixa de ferramentas tem as opções realizar medições ou } \\
\text { angulações, segundo a escala de }\end{array}$ & $80 \%$ & $20 \%$ & $* \mathrm{p}<0,001$ \\
\hline $\begin{array}{l}\text { No "corte em curva", em ferramentas possui um ícone que } \\
\text { possibilita criar a arcada manualmente para obtenção de uma vista } \\
\text { panorâmica. Para isso seleciona a área que deseja de acordo com a } \\
\text { vista 3D no plano axial (eixo roxo) }\end{array}$ & $90 \%$ & $10 \%$ & $* \mathrm{p}<0,001$ \\
\hline $\begin{array}{l}\text { O roxo corresponde a vista coronal, possibilitando movimentos no } \\
\text { sentido anteroposterior }\end{array}$ & $85 \%$ & $15 \%$ & $* \mathrm{p}<0,001$ \\
\hline $\begin{array}{l}\text { O software possibilita a aquisição de imagens em quais tipos de } \\
\text { cortes }\end{array}$ & $85 \%$ & $15 \%$ & $* \mathrm{p}<0,001$ \\
\hline
\end{tabular}

$* \mathrm{p}<0,05$ diferença estatisticamente significante; $\mathrm{n} / \mathrm{a}$ : não se aplica 
Tabela 4 - Quanto ao material de apoio disponibilizado

\begin{tabular}{lccc}
\hline \multicolumn{1}{c}{ Pergunta } & \multicolumn{3}{c}{ Respostas (\%) } \\
\cline { 2 - 4 } & Sim & Não & Valor de p \\
\hline $\begin{array}{l}\text { Você considera que as imagens existentes no } \\
\text { treinamento contribuíram na sua aprendizagem? }\end{array}$ & $100 \%$ & $0 \%$ & $\mathrm{n} / \mathrm{a}$ \\
$\begin{array}{l}\text { A forma como era disposto o conteúdo facilitou o } \\
\text { processo de ensino-aprendizagem? }\end{array}$ & $93 \%$ & $7 \%$ & $\mathrm{p}<0,001$ \\
$\begin{array}{l}\text { O Formato dos tutoriais disponibilizados no AVA } \\
\text { métodos especializados por imagem - Diagnóstico }\end{array}$ & $100 \%$ & $0 \%$ & $\mathrm{n} / \mathrm{a}$ \\
$\begin{array}{l}\text { Oral (AVA-DO) estimulou a aprendizagem? } \\
* \mathrm{p}<0,05 \text { diferença estatisticamente significante n/a: não se aplica }\end{array}$ & & \\
\end{tabular}

A partir da instituição da educação a distância no Brasil, desde século XX tem sido permitido às universidades ofertar cursos que permitem o desenvolvimento cognitivo por meio de atividades não presenciais, ou seja, sem a presença física de um professor. Com essa proposta, os educadores podem aplicar uma gama maior de recursos de aprendizagem, planejando atividades virtuais e/ou presenciais levando em consideração as limitações e potencialidade que cada modalidade apresenta ${ }^{21}$.

$\mathrm{O}$ ambiente virtual de aprendizagem (AVA) aparece então como um grande aliado ao ensino a distância, apresentando-se como uma proposta viável, já que permite que o professor customize o ambiente de trabalho para as tarefas que necessita, sem precisar de linguagem computacional para isso e que o usuário manipule o programa contando apenas com os conhecimentos básicos em informática. A grande utilidade do AVA é a possibilidade de desenvolvimento de atividades online, envolvendo recursos de telecomunicação e informática suficientes para auxiliar na construção do conhecimento $^{22}$. O ambiente virtual de aprendizagem, nos cursos de odontologia, traz a oportunidade do uso de ferramentas tecnológicas, de modo a se estabelecer uma adequada interação entre o professor e o aluno participando ativamente das atividades do grupo, em discussões de tópicos relevantes, discussões que têm como objetivo comum a transformação de suas ações através de reflexões sobre a prática pedagógica $^{16}$. A plataforma Moodle, foi desenvolvida em 1999 pelo australiano Martinn Dougiamas e amplamente disseminada pelo mundo. O objetivo de sua criação é viabilizar o acesso a aprendizagem à distância através de comunidades de usuários, valendo-se de discurso colaborativo em suas interações ${ }^{16}$. A plataforma se destaca pelas suas ferramentas de comunicação, criação e administração de componentes de aprendizagem, podendo ainda ser baixada, utilizada e/ou modificada por qualquer pessoa em qualquer lugar do mundo, contribuindo não somente para o EaD como também para o ensino presencial $^{23}$.

Nesse contexto, surge o B-Learning, que é a mais atual abordagem em educação virtual, que vai combinar o uso do ensino a distância online com o ensino presencial em sala de aula, ofertando grande vantagem e melhorando o potencial do ensino. Existem vários tipos de $B$ Learning, e estes vão depender do equilíbrio das abordagens presenciais e à distância, como por exemplo, o material didático de apoio online poder ser entregue antes ou depois do aluno participar das aulas presenciais ${ }^{14}$. Uma 
consequência do B-Learning é que as atividades passam a se posicionar em espectros contínuos no espaço real/virtual, no tempo síncrono/ assíncrono, e na interatividade passivo/ interativo $^{22}$. $\mathrm{O}$ uso das novas tecnologias aplicadas à educação enfrenta o grande desafio que é motivar o aluno a buscar o conhecimento, mesmo estando fora da sala de aula.

Os resultados obtidos no presente estudo apontam que a participação dos alunos no AVA favoreceu o processo de aprendizagem. Todos os 13 voluntários consideraram que as imagens existentes contribuíram na sua aprendizagem, em adição aos estudantes que consideraram que a disposição do conteúdo facilitou o processo de ensino-aprendizagem, e que o formato dos materiais disponibilizados AVA-DO estimulou a aprendizagem, corroborando os resultados obtidos no trabalho de Pahinis et al. ${ }^{14}$ no qual a maioria dos estudantes manifestaram satisfação fizeram satisfeitos com o curso online proposto. $\mathrm{O}$ estudo de Cruz et al. $^{24}$ também encontrou alto nível de satisfação dos estudantes com relação à experiência de aprender por meio da $\mathrm{EaD}$ via plataforma Moodle.

Analisando o percentual de ganho de aprendizado do estudante a partir das perguntas específicas do curso online sobre instalação e uso do software, constatou-se que todas as questões tiveram mais $80 \%$ de acertos com diferença estatística significante em todas as perguntas ( $\mathrm{p}<0,05)$, indicando ganho no aprendizado, em conformidade com análise feita por Haddad et $a l .{ }^{25}$, que concluíram que o uso de curso a partir de AVA foi efetivo e contribuiu para melhorar os conhecimentos e habilidades dos profissionais. No entanto no estudo Salajan et al. ${ }^{26}$ não foram encontrados dados conclusivos sobre a efetividade do processo de ensino-aprendizagem das aplicações digitais utilizadas.

Quanto à acessibilidade ao AVA-DO, observamos que houve dificuldade por parte dos alunos em acessar o ambiente virtual, pois $43 \%$ dos alunos responderam a opção nunca, contrastando com o trabalho de Jorge ${ }^{29}$, o qual confirma que sempre o aluno tem facilidade no acesso, ainda nesse quesito pode-se observar que não houve grande disparidade entre as respostas, ficando às vezes e sempre com $20 \%$ das escolhas. No que diz respeito à facilidade em acessar os conteúdos expostos, 58\% dos alunos responderam nunca, mais uma vez indo de encontro aos resultados obtidos no estudo de Jorge ${ }^{27}$, a análise dos alunos sobre a possibilidade de fazer os downloads no AVA-DO, 47\% dos voluntários indicaram a opção sempre. Os nossos resultados, também fazem oposição ao trabalho de Magagnini $^{28}$, no qual houve avaliação positiva dos conteúdos e atividades expostas em ambiente virtual de aprendizagem.

No quesito navegabilidade $50 \%$ dos estudantes indicaram que nunca tiveram facilidade em encontrar as informações desejadas, havendo empate com a opção às vezes. A opção sempre foi selecionada por $29 \%$ dos participantes. Na questão facilidade de navegação entre as diversas seções/aulas do treinamento, observamos uma disparidade entre os resultados: $50 \%$ dos alunos apontam nunca ter facilidade enquanto que $36 \%$ relatam ter sempre a facilidade em navegar pela plataforma, o que está de acordo com o estudo de Jorge ${ }^{29}$, no qual se constatou $40 \%$ de facilidade de navegação no AVA. Sendo assim, esses resultados expõem a dificuldade do estudante em manusear a plataforma Moodle, encontrando obstáculos na navegabilidade e acessibilidade da ferramenta.

Portanto, verificamos que o AVA-DO, contribuiu significativamente para o enriquecimento do conhecimento do aluno, confirmando que a educação a distância pode complementar o ensino presencial com influências positivas no processo de aprendizagem, garantindo a eficácia do Blended Learrnig no panorama educacional. 
No entanto, o presente estudo também revelou aspectos negativos quanto à pouca familiaridade dos alunos com a estrutura da plataforma, evidenciando a necessidade de aprimoramentos para facilitar e viabilizar o uso das tecnologias de informação e comunicação, em favor da aprendizagem.

\section{CONCLUSÃO}

De acordo com o exposto anteriormente, podemos concluir que a utilização do B-learning, por meio do ambiente virtual de aprendizagem favoreceu o processo de aprendizagem dos estudantes de graduação, consistindo em mais um cenário de aprendizagem para a Odontologia.

\section{ABSTRACT \\ Evaluation and development of a virtual learning environment in the teaching of Oral Diagnosis through blended learning}

Blended Learning (B-learning) combines the use of distance learning with face-to-face teaching in the classroom. The Moodle platform is a Learning Management System (LMS), which works based on the construction of learning from interaction, and also offers tools that favor the integration of face-to-face activities with distance activities, but easy to use. The objective of this study was to evaluate and expand learning scenarios by creating a virtual environment as support teaching material, with the aim of transforming it into a tool in the education process for students. For this purpose, 13 undergraduate students were selected at the Federal University of Sergipe. At the end of the course, the volunteers were submitted to a questionnaire to evaluate the AVA specialized imaging methods - oral diagnosis (AVA-DO), regarding the support material available in the environment, as well as accessibility and usability and the gain of knowledge of the student after the experience in distance education. The evaluation of the student's learning was made through a questionnaire regarding the material taught. All questions had
$80 \%$ correctness. The results also expose the student's difficulty in handling Moodle, finding obstacles in the usability and accessibility of the tool. It is concluded that the use of the virtual learning environment favored the learning process of undergraduate students, consisting of yet another learning scenario for dentistry.

Descriptors: Education. Distance, Learning. Radiology.

\section{REFERÊNCIAS}

1. Cardoso C, Giotto E, Karsburg A, Steinmetzk C, Schio F. Gestão rural no contexto da educação à distância. [Acesso em 20 fev. 2016]. Disponível em: https://ead.ufsc.br/seminario2012/files/2012 104/ Anais-versãopreliminar-.pdf.

2. El Tantawi MM, El Kashlan MK, Saeed YM. Assessment of the efficacy of second life, a virtual learning environment, in dental education. J Dent Educ. 2013;77(12):163952.

3. Ogliari CL, DE Souza MV. EaD e os Desafios das Novas Tecnologias: LabMídia da UFSC - Campus Araranguá, uma experiência de apoio à educação em rede. 2012. [Acesso em 20 fev. 2016]. Disponível em: http://www.labmidiaeconhecimento. ufsc.br/files/2012/07/EaD-e-os-Desafios-dasNovas-Tecnologias-LabM\%C3\%AD dia-daUFSC-Campus-Ararangu $\% \mathrm{C} 3 \% \mathrm{~A} 1-\mathrm{uma}-$ experi\%C3\%AAncia-de-apoio-\%C3\% A0educa\%C3\%A7\%C3\%A3o-em-rede.pdf.

4. Belloni M L. Educação à distância. Campinas. SP: Autores Associados, 2003.

5. Bissoli CF, Takeshita WM, Castilho JCM. Digitalização de imagens em radiologia: uma nova visão de futuro. Rev Odonto 2007;15(30):34-9.

6. Takeshita WM, Iwaki LCV, Da Silva MC, Sábio S, Albino, PRF. Comparison of periapical radiography with cone beam computed tomography in the diagnosis of 
vertical root fractures in teeth with metallic post. J Conserv Dent. 2014;17(3):225-9.

7. Takeshita WM, Dos Santos LRA, Castilho J.C.M, Medici-Filho E, Moraes LC, Sannomiya, E.K. An investigation of the optical density of composite resin using digital radiography. Cienc Odontol Bras. 2004;7(2):6-11.

8. Veersteeg $\mathrm{CH}$, Sanderink GCH, Van Der Stelp PF. Efficacy of digital intra-oral radiology in clinical dentistry. $\mathrm{J}$ Dent. 1997;25(3):215-24.

9. Chen SK, Chiang TC. Digitizing of radiographs with a rollertype CCD scanner. Oral Surg Oral Med Oral Pathol Oral Radiol. 1997;83(6):719-24.

10. Medici-Filho E, Castilho JCM, Moraes LC, Takeshita WM. Sistema radiográfico digital: aspectos importantes na aquisição dos principais sistemas. Rev ABO Nac. 2006;14(3):152-55.

11. Amaya M. O mito do megapixel. [Acesso em 05 abr. 2014]. Disponível em: http://www.photoshopcreative.com.br/artigos/a q0-124-7102-1-o+mito+do+megapixel.html.

12. Saliba NA, Moimaz SAS, Prado RL, Garbin CAS. Percepção do cirurgião-dentista sobre formação profissional e dificuldades de inserção no mercado de trabalho. Rev Odontol UNESP. 2012;41(5):297-304.

13. Freitas VP, Carvalho RB, Gomes MJ, Figueiredo MC, Silva DDF. Mudança no processo ensino- aprendizagem nos cursos de graduação em odontologia com utilização de metodologias ativas de ensino e aprendizagem. Rev Fac Odontol RFO. 2009;14(2):163-67.

14. Pahinis K, Stokes CW, Walsh TF, Tsitrou E, Cannavina G. A blended learning course taught to different groups of learners in a dental school: follow-up evaluation. J Dent Educ. 2008;72(9):1048-57.
15. Borak S, S. Medicina bucal: tratamento clínico-cirúrgico das doenças bucomaxilofaciais. São Paulo: Artes Médicas, 2011.

16. Cunha-Araújo IMZ, Salazar-Silva JR, D’Assunção FLC, Melo ABP. Avaliação da percepção dos alunos da disciplina de endodontia sobre o uso do ambiente virtual de aprendizagem (Moodle). Uso do questionário de auto-avaliação Colles. Rev ABENO. 2012;12(2):163-69.

17. Arevalo RC, Bayne E, Beeley JA, Brayshaw CJ, Cox MJ, Donaldson NH. Framework for E-Learning Assessment in Dental Education: A Global Model for the Future. J Dent Educ. 2013;77( 2):564-75.

18. Gottlieb R, Lanning S, Gunsolley JC, Buchanan JA. Faculty impressions of dental students' performance with and without virtual reality simulation. J Dent Educ. 2011;75(11): 1443-51.

19. Moazami F, Bahrampour E, Azar M.R., Jahedi F, Moattari M. Comparing two methods of education (virtual versus traditional) on learning of Iranian dental students: a post-test only design study. BMC Med Educ. 2014;14:45.

20. Freitas VP, Carvalho RB, Gomes MJ, Figueiredo MC, Faustino-Silva, D.D. Mudança no processo ensinoaprendizagem nos cursos de graduação em odontologia com utilização de metodologias ativas de ensino e aprendizagem. Rev Fac Odontol RFO. 2009;14(2):163-7.

21. Alencar CJF. Impacto das novas tecnologias de informação e comunicação, através do blended learning, aplicadas aos graduandos em odontopediatria [dissertação]. São Paulo: Faculdade de Odontologia da Universidade de São Paulo. Programa de Pós-Graduação em Ciências Odontológicas. 2012

22. Dotta EA, Garcia PPNS, Candido LM. Elaboração de um curso interativo voltado 
ao aprendizado de um sistema aplicativo em Odontologia, utilizando a plataforma Moodle. Rev Odontol Univ Cid São Paulo. 2012;24(1):6-14.

23. Maciel DT, Soares W, Amaral E. Moodle platform for online tutoring during internship. [Acesso em 20 fev. 2016]. Disponível em: http://onlinelibrary.wiley. com/doi/10.1111/j.1365-2923.2009.03462.X/ epdf.

24. Cruz AD, Costa JJ, Almeida SM. Distance learning in dental radiology: immediate impact of the implementation. Braz Dent Sci 2014;17(4):90-7.

25. Haddad AE, Skelton-Macedo MC. Teleodontologia na formação dos profissionais de saúde. [Acesso em 20 fev. 2016]. Disponível em: http://www.teles saude.uerj.br/resource/goldbook/pdf/12.pdf.

26. Salajan FD, Mount, GJ. University of Toronto's Dental School Shows "New Teeth": Moving Towards Online Instruction. J Dent Educ. 2008;72(5):532-42
27. Jorge LS. Avaliação de ambiente virtual de aprendizagem na modalidade semipresencial no ensino de graduação em odontologia da UFC. Dissertação [Mestrado em Odontologia]. Universidade Federal do Ceará (UFC); 2013.

28. Magagnini AC, Scabora JE, Esquisatto MAM. Utilização de recursos didáticos digitais na disciplina semipresencial de Imaginologia Odontológica para alunos de Mestrado Profissional. [Dissertação] Mestrado Profissional em Odontologia. Centro Universitário Hermínio Ometto de Araras; 2014.

Correspondência para:

Ana Cristina Zuzarte Ferreira Santos e-mail:anaczfs@gmail.com Av Franklin de Campos Sobral, 2085. 49027-000 Jardim Jussara - Aracaju/SE 